\title{
Similarity Criteria of Water Drive Physical Simulation of Pressure-Sensitive Fractured Reservoirs
}

\author{
Nie Bin $\left(\mathbb{D},{ }^{1}\right.$ Gu Shaohua $\mathbb{D}^{2},{ }^{2}$ and Zeng Sijia $\mathbb{( D}^{1}$ \\ ${ }^{1}$ School of Petroleum Engineering, Yangtze University, Wuhan, Hubei 430100, China \\ ${ }^{2}$ Petroleum Exploration \& Production Research Institute, SINOPEC, Beijing 100083, China \\ Correspondence should be addressed to Nie Bin; niebin19880918@163.com
}

Received 19 April 2021; Revised 4 July 2021; Accepted 2 August 2021; Published 17 August 2021

Academic Editor: Hao Xiong

Copyright ( 2021 Nie Bin et al. This is an open access article distributed under the Creative Commons Attribution License, which permits unrestricted use, distribution, and reproduction in any medium, provided the original work is properly cited.

\begin{abstract}
A mathematical equation of water drive physical simulation of pressure-sensitive fractured reservoirs was established based on previous research results. In this study, the similarity criteria of water drive physical simulation of pressure-sensitive fractured reservoirs were derived according to the similarity theory. First of all, based on the three-dimensional differential equation of rock mechanics, a dimensionless analysis was conducted to determine the similarity relationship between the displacement of oil by water of pressure-sensitive fractured reservoirs, the similarity criterion was obtained, and the similarity criteria were formed. Secondly, according to the similarity criterion, the similar relationship between the stress-strain fields of the real object and the simulated object was worked out. Thirdly, the finite element software COMSOL Multiphysics was applied to model and calculate the multifield coupling process in the percolation of pressure-sensitive fractured reservoirs, verifying the correctness of the established similarity criteria and similarity relationship. The verifying results shows that the similarity between the physical model and the actual model can be realized by magnifying the geometric size $N$ times in a certain direction and adjusting the load and boundary conditions according to the similarity principle, which can be used for the design of the pressure-sensitive fractured reservoir simulation model for a physical indoor test.
\end{abstract}

\section{Introduction}

The reservoir simulation has two types of approach, numerical reservoir simulation and physical reservoir simulation. The numerical stimulation predicts the flow of liquids in the reservoir (oil, gas, and water) through porous media once the production starts. These real-time simulations are studied using computer models which calibrate the data based on numerical equations and predict the behavior of the reservoir once the production starts. The other method is physical reservoir simulation, which is a quantitative $3 \mathrm{D}$ model build by researchers in the laboratory to simulate the actual reservoir. Considering reservoir, wellbore, and fluid, the quantitative $3 \mathrm{D}$ physical reservoir simulation method is established based on the similarity criteria. To further find out the variation pattern of fracture permeability during the water drive development of pressure-sensitive fractured reservoirs, it is necessary to carry out three-dimensional quantitative physical simulation, which is a method of reservoir simulation by building an indoor physical model to simulate the field reservoir. Since the reservoir scale is too large, the currently feasible physical simulation methods are mainly realized by building indoor physical models. However, the results obtained from indoor physical experiments cannot be directly applied to the actual situation, and it is of necessity to match the indoor experimental results to the actual development process of reservoirs through the similarity criteria, so as to guide the actual development of oil and gas fields. Similarity criteria refer to a theory that explains all types of similar phenomena and principles in nature and engineering science. Similarity criteria, based on the three theorems of similarity, are used to guide the design of models and the processing and generalization of related experimental data. The role of similarity criteria must explain theoretically the following contents: the nature of similar phenomena, the application of similar phenomena, and the conditions to achieve similarity. To answer these questions, analysis and derivation must be carried out according to the three 

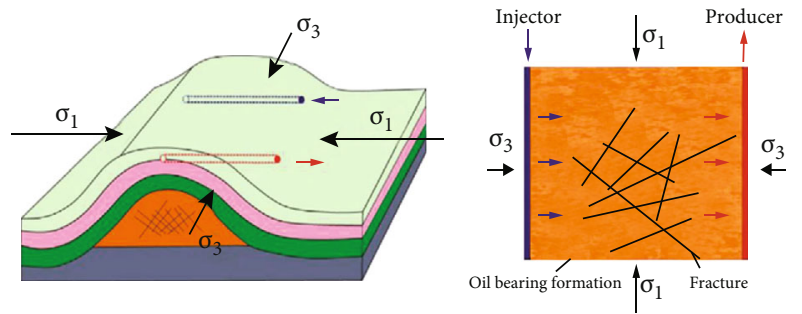

FIGURE 1: A sketch map of the flow in a fractured reservoir with in situ stress [19].

theorems of similarity based on the basic principles of simulation experiments.

In recent years, many scholars have carried out research on the similarity theory related to the development of all kinds of reservoirs, achieving many results. Firstly, water drive sandstone reservoirs are the most typical reservoir and many researchers established many similarity criteria for this kind of reservoir, such as Bai and Zhou, Ding et al., $\mathrm{Gu}$ et al., Kong and Chen, Li, Bai et al., and Zhou [1-6]. For this type of reservoirs, the similarity criteria just need to take few parameters into consideration, such as the permeability, the porosity, and the characteristic length of the model. Then, they carried out the corresponding physical simulation experiments. Shen et al. and Teng et al. [7, 8] extended the study on similarity criteria to tight gas reservoirs, considering the pressure-sensitive characteristics to some extent by establishing the empirical relationship between pressure and permeability. On comparison of single porous media, the dual porous media with fractures are more complex, because the property and flow in the fracture system and the exchange between fracture and matrix need to be put into consideration. According to the percolation equation of double media, Shi et al. and Ding et al. [9, 10] established the similarity criteria of water drive reservoirs for carbonate gas reservoirs. As the research goes deep, some scholars have also studied the similarity criteria for physical simulation of special types of oil and gas reservoirs such as thermal recovery of heavy oil [11], polymer flooding [12$14]$ and gas hydrate $[15,16]$, and shale gas [17] and coalbed-methane [18].

However, there is currently no similarity theory applied to the simulated development of pressure-sensitive fractured reservoirs, making it difficult to solve the problem of crossscale modeling of reservoir development, as Figure 1. Because more coupling fields need to be considered simultaneously, such as the stress-strain field, the flow pressure field, and the fracture-matrix permeability field, no similarity criterion number for this type of reservoir have not been reported before. Hence, to solve this problem, a method of establishing the similar model for the stress-strain field of the pressuresensitive fractured medium was proposed based on the similarity principle in this study. In other words, a similar model with magnified thickness was set up to study the percolation of stress-sensitive fractured anisotropic reservoirs. Since the dimensions of all directions of the similar model are of the same order of magnitude, when meshing the elements, the number of elements in the finite element model can be effec- tively reduced and the amount of calculation can be reduced while ensuring the appropriate aspect ratio of the elements. In this study, the similarity criteria and similarity relationship of the stress-strain field were derived and established, and the correctness of similarity criteria and similarity relationship was verified by the finite element software, thereby laying a solid theoretical foundation for guiding the physical simulation of multiphysical field coupling in pressuresensitive fractured anisotropic reservoirs. This work is aimed at providing a method to design an experimental model in the lab to simulate the flow and stress-strain performance of an actual fractured reservoir precisely.

\section{Similarity Criteria of Saturated Fluid Matrix considering Pressure Dependence}

2.1. Stress-Strain Model of Saturated Fluid Matrix. The fluid percolation model in underground porous media mainly includes the matrix field flow equation, fracture field flow equation, and stress field variation equation. According to the study by $\mathrm{Gu}$ et al. [19], the equations were established, and the equation analysis method was used to deduce the similarity criteria of pressure-sensitive fractured reservoirs.

2.1.1. Physical Equation. First of all, according to Biot's elastic porous medium theory, the effective stress equation of the medium should be expressed as follows:

$$
\left[\begin{array}{c}
\sigma_{x} \\
\sigma_{y} \\
\sigma_{z} \\
\tau_{y z} \\
\tau_{x z} \\
\tau_{x y}
\end{array}\right]=C\left[\begin{array}{c}
\varepsilon_{x} \\
\varepsilon_{y} \\
\varepsilon_{z} \\
\gamma_{y z} \\
\gamma_{x z} \\
\gamma_{x y}
\end{array}\right]+\alpha\left[\begin{array}{c}
p \\
p \\
p \\
0 \\
0 \\
0
\end{array}\right]
$$

Equation (1) can also be expressed as

$$
\sigma=C \varepsilon+\alpha p I
$$

where $\sigma=\left\{\sigma_{x}, \sigma_{y}, \sigma_{z}, \tau_{y z}, \tau_{x z}, \tau_{x y}\right\}^{T}$ is the Cauchy stress tensor, $\varepsilon=\left\{\varepsilon_{x}, \varepsilon_{y}, \varepsilon_{z}, \gamma_{y z}, \gamma_{x z}, \gamma_{x y}\right\}^{T}$ is the strain tensor, $\alpha$ stands for the Biot coefficient, $p$ is pore pressure of the fluid, and array $I$ is expressed as $\{1,1,1,0,0,0\}^{T}$. In Equation (2), all the coefficients in elastic equation $C$ must be measured in the percolation state with constant pore pressure.

$$
C=\left[\begin{array}{cccccc}
c_{11} & c_{12} & c_{13} & c_{14} & c_{15} & c_{16} \\
& c_{22} & c_{23} & c_{24} & c_{25} & c_{26} \\
& & c_{33} & c_{34} & c_{35} & c_{36} \\
& & & c_{44} & c_{45} & c_{46} \\
& & & & c_{55} & c_{56} \\
& & & & & c_{66}
\end{array}\right] .
$$




\subsubsection{Geometric Equation.}

$$
\left\{\begin{array}{l}
\varepsilon_{x}=\frac{\partial u}{\partial x}, \gamma_{x y}=\frac{\partial u}{\partial y}+\frac{\partial v}{\partial x} \\
\varepsilon_{y}=\frac{\partial v}{\partial y}, \gamma_{y z}=\frac{\partial v}{\partial z}+\frac{\partial w}{\partial y} \\
\varepsilon_{z}=\frac{\partial w}{\partial z}, \gamma_{x z}=\frac{\partial u}{\partial z}+\frac{\partial w}{\partial x}
\end{array}\right.
$$
lows:

Equation (4) can be written into the matrix form as fol-

$$
\varepsilon=L U
$$

where $U=[u, v, w]^{T}$ is an array about the displacements in the $x, y$, and $z$ directions.

\subsubsection{Equilibrium Equation.}

$$
\left\{\begin{array}{l}
\frac{\partial \sigma_{x}}{\partial x}+\frac{\partial \tau_{y x}}{\partial y}+\frac{\partial \tau_{z x}}{\partial z}+f_{x}=0 \\
\frac{\partial \tau_{x y}}{\partial x}+\frac{\partial \sigma_{y}}{\partial y}+\frac{\partial \tau_{z y}}{\partial z}+f_{y}=0 \\
\frac{\partial \tau_{x z}}{\partial x}+\frac{\partial \tau_{y z}}{\partial y}+\frac{\partial \sigma_{z}}{\partial z}+f_{z}=0
\end{array}\right.
$$

Equation (6) can be written into a matrix form as follows:

$$
L^{T} \sigma+\bar{f}=0
$$

where $f=\left\{f_{x}, f_{y}, f_{z}\right\}^{T}$ represents an array about the displacements in the $x, y$, and $z$ directions. Matrix $L$ is a partial differential operator matrix that can be expressed as

$$
L=\left[\begin{array}{ccc}
\frac{\partial}{\partial x} & 0 & 0 \\
0 & \frac{\partial}{\partial y} & 0 \\
0 & 0 & \frac{\partial}{\partial z} \\
0 & \frac{\partial}{\partial z} & \frac{\partial}{\partial y} \\
\frac{\partial}{\partial z} & 0 & \frac{\partial}{\partial x} \\
\frac{\partial}{\partial y} & \frac{\partial}{\partial x} & 0
\end{array}\right]
$$

According to Equations (2), (5), and (8), it can be further expressed as

$$
L^{T}=(\mathrm{CPU}-\alpha p I)+\bar{f}=0 .
$$

\subsubsection{Boundary Conditions}

(1) Stress Boundary. On boundary $\Gamma$, the boundary conditions of the stress field can be expressed as follows:

$$
\left\{\begin{array}{l}
p_{\Gamma x}=n_{x} \sigma_{x}+n_{y} \tau_{y z}+n_{z} \tau_{z x}+\zeta_{x} u \\
p_{\Gamma y}=n_{x} \tau_{x y}+n_{y} \sigma_{y}+n_{z} \tau_{z y}+\zeta_{y} u \\
p_{\Gamma z}=n_{x} \tau_{x z}+n_{y} \tau_{y z}+n_{z} \sigma_{z}+\zeta_{z} u
\end{array}\right.
$$

which can be written into a matrix form

$$
P_{\Gamma}=n \sigma+\bar{Z} U
$$

By substituting the stress equation (2) of the matrix into Equation (11), the following equation can be obtained:

$$
p_{\Gamma}=n(\mathrm{CLU}-\alpha p I)+\bar{Z} U \text {, }
$$

where matrix $\bar{Z}$ is a matrix of the elastic boundary limiting coefficient which can be expressed as

$$
\bar{Z}=\left[\begin{array}{ccc}
\zeta_{x} & 0 & 0 \\
0 & \zeta_{y} & 0 \\
0 & 0 & \zeta_{z}
\end{array}\right]
$$

In Equation (12), $n$ refers to

$$
n=\left[\begin{array}{cccccc}
n_{x} & 0 & 0 & n_{y} & 0 & n_{z} \\
0 & n_{y} & 0 & n_{x} & n_{z} & 0 \\
0 & 0 & n_{z} & 0 & n_{y} & n_{x}
\end{array}\right]
$$

where $n_{x}, n_{y}$, and $n_{z}$ denote the direction cosines of the outer boundary in the normal direction.

(2) Displacement Boundary. On boundary $\Gamma$, the displacement boundary of the stress field can be expressed as follows:

$$
u=u_{\Gamma}, v=v_{\Gamma}, w=w_{\Gamma} .
$$

By introducing the potential equation $\psi$, Equation (15) can be expressed as

$$
u=\frac{\partial \psi}{\partial x}, v=\frac{\partial \psi}{\partial y}, w=\frac{\partial \psi}{\partial z}
$$

It can be further expressed in the matrix form

$$
U=A \bar{\psi}
$$

where operator $\mathrm{A}$ is expressed as

$$
A=\left[\frac{\partial}{\partial x} \frac{\partial}{\partial y} \frac{\partial}{\partial z}\right]^{T}
$$

By substituting Equation (17) into Equations (9), (12), 
and (17), the equilibrium equation can be transformed into

$$
L^{T}=n(\mathrm{CLA} \psi-\alpha p I)+\bar{f}=0 .
$$

On boundary $\Gamma$, the boundary conditions of the stress can be expressed as follows

$$
p_{\Gamma}=n(\mathrm{CLA} \psi-\alpha p I)+\bar{Z} U .
$$

At this time, the displacement boundary conditions are

$$
\overline{U_{\Gamma}}=A \psi .
$$

2.2. Derivation of Similarity Criteria. By analyzing the equations in the appendix, it was found that to determine the unique dimensionless potential function $\Psi, 43$ mutually independent similarity criteria were needed. Therefore, 43 mutually independent parameters were selected from $X, Y$, $Z, P, P_{x}, P_{y}, P_{z}, F_{x}, F_{y}, F_{z}, U_{\Gamma}, V_{\Gamma}$, and $W_{\Gamma}$ and various nondimensional coefficients as the similar criteria of the pressure-sensitive medium, as shown in Table 1.

\section{Similarity Criteria of Saturated Fluid Fracture considering Pressure Dependence}

According to the study conducted by Gu et al. [19], fracture aperture can be calculated based on Equation (22),

$$
\begin{aligned}
& d_{f}= d_{n-0}-\frac{d_{n \max }}{1+\left(\left(K_{n 0} \cdot d_{n \max }\right) / \sigma_{n}\right)}+\min \left(d_{n-s d}, u_{c s}\right), \\
& \sigma_{n}^{\prime}= \begin{cases}\sigma_{s 0} \leq \sigma_{s} \leq \sigma_{c} & \sigma_{n}, \\
\sigma_{c} \leq \sigma_{s} \leq \sigma_{s-\text { peak }} & \frac{\sigma_{n}-d_{n-s d}}{K_{n}\left(\sigma_{n}\right)}, \\
\sigma_{s-\text { peak }}=\sigma_{s} & \frac{\sigma_{n}-d_{n-s d}}{K_{n}\left(\sigma_{n}\right)},\end{cases} \\
& d_{n-s d}= \begin{cases}\sigma_{s 0} \leq \sigma_{s} \leq \sigma_{c} & 0, \\
\sigma_{c} \leq \sigma_{s} \leq \sigma_{s-\text { peak }} & \frac{\left(\sigma_{s}-\sigma_{c}\right) \cdot \tan (\psi)}{K_{s}}, \\
\sigma_{s-\text { peak }}=\sigma_{s} & \frac{\left(\sigma_{s-\text { peak }}-\sigma_{c}\right) \cdot \tan (\psi)}{K_{s}}+\frac{\left(\sigma_{n}-\sigma_{n-\text { peak }}\right)}{\left(K_{n}\left(\sigma_{n}\right) \cdot \tan (\psi)\right)} .\end{cases}
\end{aligned}
$$

To nondimensionalize Equation (22), the following scales were introduced:

Length scale: $\overline{d_{f}}$ denotes the unit length of fracture aperture, $\overline{d_{n-0}}$ is the unit length of fracture aperture at the initial moment, $\overline{d_{n \text { max }}}$ represents the unit length of maximum normal fracture aperture, $\overline{d_{n-s d}}$ is the unit length of fracture aperture caused by shear expansion, and $\overline{u_{c s}}$ is the unit length of the ultimate shear expansion, with $[L]$ being the dimension.

Stress scale: $\overline{\sigma_{n}{ }^{\prime}}$ is the actual unit normal stress, $\overline{\sigma_{n}}$ denotes the total unit normal stress, $\overline{\sigma_{s}}$ represents the unit shear stress, $\overline{\sigma_{s 0}}$ is the initial unit shear stress, $\overline{\sigma_{s-\text { peak }}}$ is the unit peak shear stress, and $\overline{\sigma_{c}}$ denotes the unit shear strength, with $\left[L^{-1} M T^{2}\right]$ being the dimension.

Rigidity scale: the initial unit normal rigidity $K_{n 0}$ was expressed as $\overline{K_{n 0}}$, the unit normal rigidity $K_{n}$ was expressed as $\overline{K_{n}}$, and the unit tangential rigidity $\mathrm{K}_{\mathrm{s}}$ was expressed as $\overline{K_{s}}$, with $\left[L^{-2} M T^{2}\right]$ being the dimension,

Tan denotes the direction cosine of the unit shear expansion angle, which is dimensionless.

The parameters were nondimensionalized as follows:
$D_{n-0}=d_{n-0} / \overline{d_{n-0}}$ is the dimensionless fracture aperture.

$D_{n-0}=d_{n-0} / \overline{d_{n-0}}$ denotes the initial dimensionless fracture aperture.

$D_{n \max }=d_{n \max } / \overline{d_{n \max }}$ is the maximum dimensionless normal fracture aperture.

$D_{n-s d}=d_{n-s d} d \overline{d_{n-s d}}$ refers to the dimensionless fracture aperture caused by shear expansion.

$U_{c s}=\mu_{c s} / \overline{\mu_{c s}}$ is the limit of dimensionless shear expansion.

$\sum_{n}^{\prime}=\sigma^{\prime}{ }_{n} / \overline{\sigma_{n}^{\prime}}$ denotes the actual dimensionless normal stress.

$\sum_{n}=\sigma_{n} / \sigma_{n}$ is the total dimensionless normal stress.

$\sum_{s}^{n}=\sigma_{s} / \bar{\sigma}_{s}$ is the dimensionless shear stress.

$\sum_{s-\text { peak }}=\sigma_{s-\text { peak }} / \overline{\sigma_{s-\text { peak }}}$ is the peak dimensionless shear stress.

$\sum_{s 0}=\sigma_{s 0} / \overline{\sigma_{s 0}}$ is the initial dimensionless shear stress.

$\sum_{c}=\sigma_{c} / \bar{\sigma}_{c}$ is the dimensionless shear strength.

$K_{n 0}^{\prime}=K_{n 0} / \overline{K_{n 0}}$ denotes the initial dimensionless normal rigidity.

$K_{n}^{\prime}=K_{n} / \overline{K_{n}}$ refers to the dimensionless normal rigidity. 


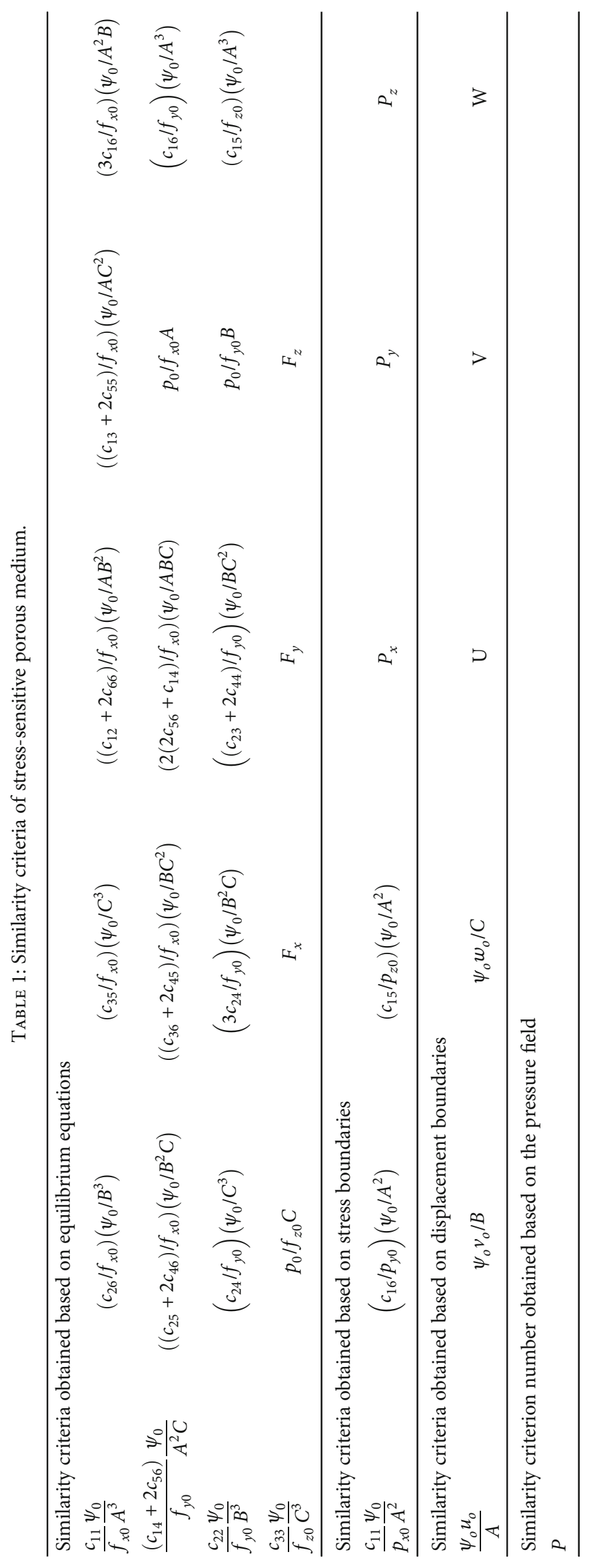




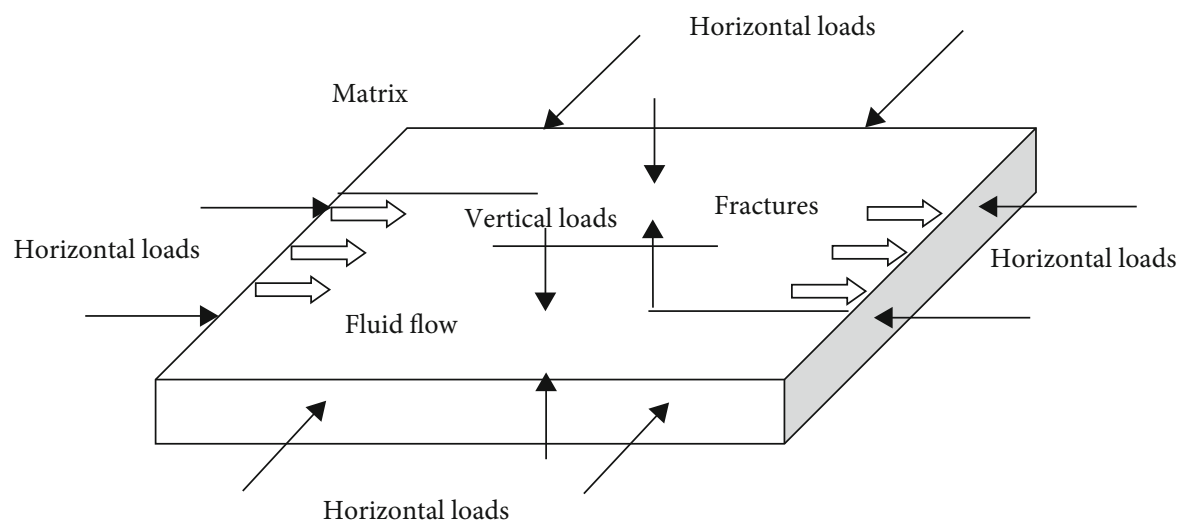

FIGURE 2: Similarity criteria model of physical simulation of water drive fractured reservoirs.

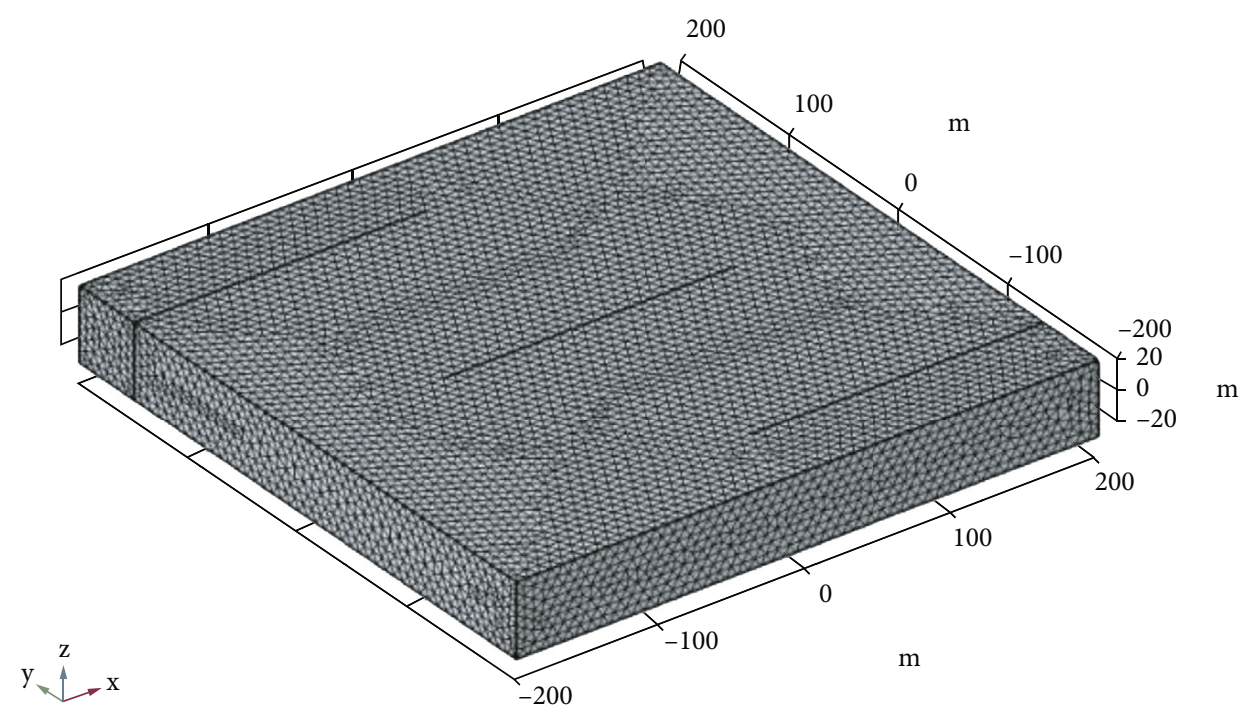

(a) Grid generation of the prototype

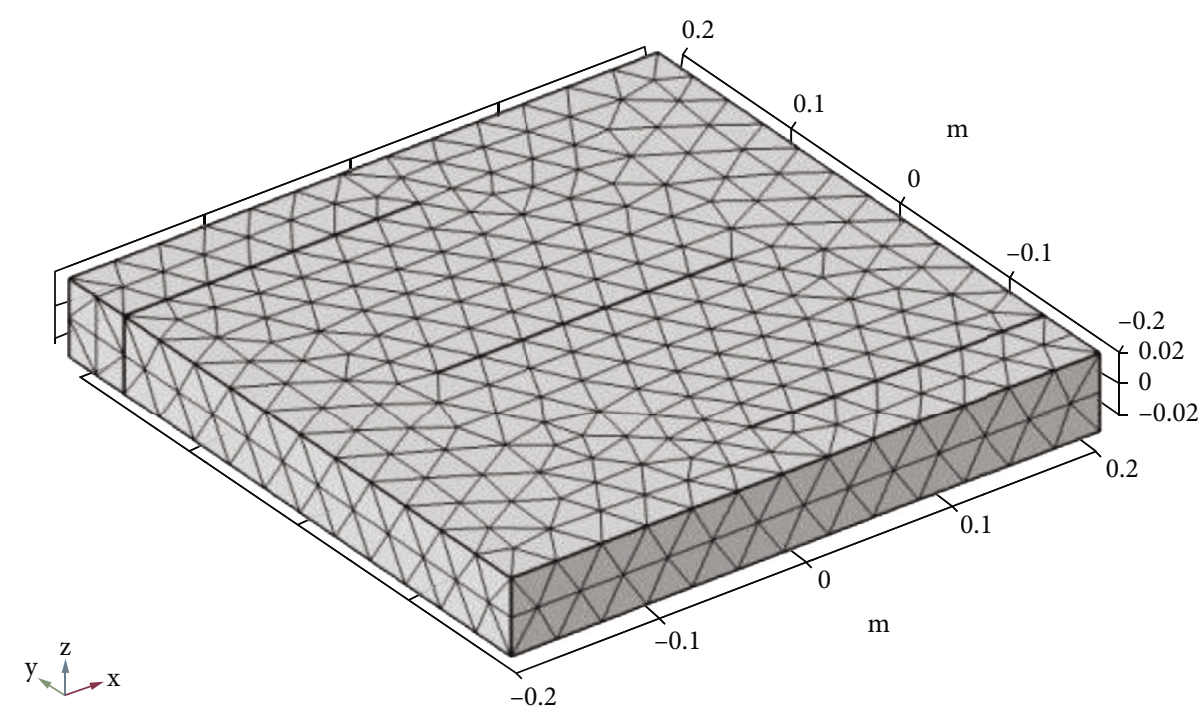

(b) Grid generation of the model

FIGURE 3: Grid generation of the prototype and the model. 
TABle 2: Physical parameters of prototype and model.

\begin{tabular}{|c|c|c|c|c|}
\hline \multirow{4}{*}{ Pressure control } & Original formation pressure $(p)$ & $10 \mathrm{MPa}$ & $10 \mathrm{MPa}$ & 1 \\
\hline & Pressure at the inlet & $15 \mathrm{MPa}$ & $10 \mathrm{MPa}$ & 1 \\
\hline & Pressure at the outlet & $5 \mathrm{MPa}$ & $5 \mathrm{MPa}$ & 1 \\
\hline & Displacement differential pressure & $10 \mathrm{MPa}$ & $1000 \mathrm{pa}$ & 1000 \\
\hline \multirow{4}{*}{ Properties of porous medium host material } & Young's modulus & $5.8 \mathrm{GPa}$ & $5.8 \mathrm{GPa}$ & 1 \\
\hline & Poisson's ratio & 0.3 & 0.3 & 1 \\
\hline & Porosity & 0.18 & 0.18 & 1 \\
\hline & Permeability & $1 \mathrm{mD}$ & $1 \mathrm{mD}$ & 1 \\
\hline \multirow{3}{*}{ Properties of the fluid } & Compressibility of the fluid & $4 e-10 \mathrm{~Pa}^{-1}$ & $4 e-10 \mathrm{~Pa}^{-1}$ & 1 \\
\hline & Density & $1000 \mathrm{~kg} / \mathrm{m}^{3}$ & $1000 \mathrm{~kg} / \mathrm{m}^{3}$ & 1 \\
\hline & Dynamic viscosity & $1 e-7 \mathrm{~Pa} \mathrm{~s}$ & $1 e-7 \mathrm{~Pa} \mathrm{~s}$ & 1 \\
\hline \multirow{2}{*}{ Boundary load } & Boundary load (in 6 directions) & $20 \mathrm{MPa}$ & $10.01 \mathrm{MPa}$ & \\
\hline & Differential pressure of boundary load & $10 \mathrm{MPa}$ & $0.01 \mathrm{MPa}$ & 1 \\
\hline Model size & Model size (length $\backslash$ width $\backslash$ height) & $400 \mathrm{~m} \backslash 400 \mathrm{~m} \backslash 50 \mathrm{~m}$ & $0.4 \mathrm{~m} \backslash 0.4 \mathrm{~m} \backslash 0.05 \mathrm{~m}$ & 1000 \\
\hline \multirow{4}{*}{ Properties of porous medium fractured material } & Load-free width of fracture & $1.5 \mathrm{~mm}$ & $1.5 \mathrm{~mm} / 31.62$ & 31.62 \\
\hline & Initial width of fracture & $1 \mathrm{~mm}$ & $1 \mathrm{~mm} / 31.62$ & 31.62 \\
\hline & Permeability of fracture & {$[1 \mathrm{~mm}]^{2} / 12$} & {$[1 \mathrm{~mm}]^{2} /(12 * 1000)$} & 1000 \\
\hline & Change of fracture width & $0.01 \mathrm{~mm}$ & $0.01 \mathrm{~mm} / 31.62$ & 31.62 \\
\hline
\end{tabular}

$K_{s}^{\prime}=K_{s} / \overline{K_{s}}$ is the dimensionless tangential rigidity.

$T^{\prime}=\tan (\psi) /$ Tan is dimensionless.
By substituting the above dimensionless parameters into Equation (40), dimensionless Equation (23) was obtained.

$$
\begin{aligned}
& D_{f}=D_{n-0}-\frac{D_{n \max }}{1+\left(\left(K_{n 0} \cdot D_{n \max }\right) / \sum_{n}^{\prime}\right)}+\min \left(D_{n-s d}, u_{c s}\right) \text {, } \\
& \Sigma_{n}^{\prime}= \begin{cases}\sum_{s 0} \leq \Sigma_{s} \leq \Sigma_{c} & \Sigma_{n} \\
\Xi_{c} \leq \Sigma_{s} \leq \Sigma_{s-\text { peak }} & \frac{\sum_{n}-D_{n-s d}}{K^{\prime}{ }_{n}\left(\Sigma_{n}\right)} \\
\sum_{s-\text { peak }}=\Sigma_{s} & \frac{\sum_{n}-D_{n-s d}}{K_{n}^{\prime}\left(\Sigma_{n}\right)}\end{cases} \\
& D_{n-s d}= \begin{cases}\sum_{s 0} \leq \Sigma_{s} \leq \Sigma_{c} & 0 \\
\Sigma_{c} \leq \Sigma_{s} \leq \Sigma_{s-\text { peak }} & \frac{\left(\Sigma_{s}-\Sigma_{c}\right) \cdot T^{\prime}}{K^{\prime}{ }_{s}}, \\
\sum_{s-\text { peak }}=\Sigma_{s} & \frac{\left(\Sigma_{s-\text { peak }}-\Sigma_{c}\right) \cdot T^{\prime}}{K^{\prime}}+\frac{\left(\Sigma_{n}-\Sigma_{n-\text { peak }}\right)}{\left(K_{n}^{\prime}\left(\Sigma_{n}\right) \cdot T^{\prime}\right)} .\end{cases}
\end{aligned}
$$

When the shear stress is in different ranges, the similarity criterion number can be different. Therefore, the similarity criterion number can be represented as follows:

When $\sum_{s 0} \leq \sum_{s} \leq \sum_{c}$, 6 similarity criteria were needed.

$$
\overline{d_{f}}, d_{\overline{n-\sigma}}, \overline{d_{n \max }}, \frac{\overline{\sigma_{n}}}{\overline{K_{n 0}} \cdot \overline{d_{n \max }}}, \overline{d_{n-s d}}, \overline{\mu_{c s}} .
$$

When $\sum_{c} \leq \sum_{s} \leq \sum_{s-\text { peak }}, 9$ similarity criteria were needed.

$$
\overline{d_{f}}, d_{\overline{n-0}}, \overline{d_{n \max }}, \overline{K_{n 0}} \cdot \overline{d_{n \max }}, \overline{\sigma_{n}}, \frac{\overline{K_{n}}}{\overline{d_{n-s d}}}, \overline{\sigma_{s 0}}, \overline{\sigma_{c}}, \frac{\overline{K_{s}}}{\overline{\mathrm{Tan}}}
$$

When $\sum_{s-\text { peak }}=\sum_{s}, 11$ similarity criteria were needed. 


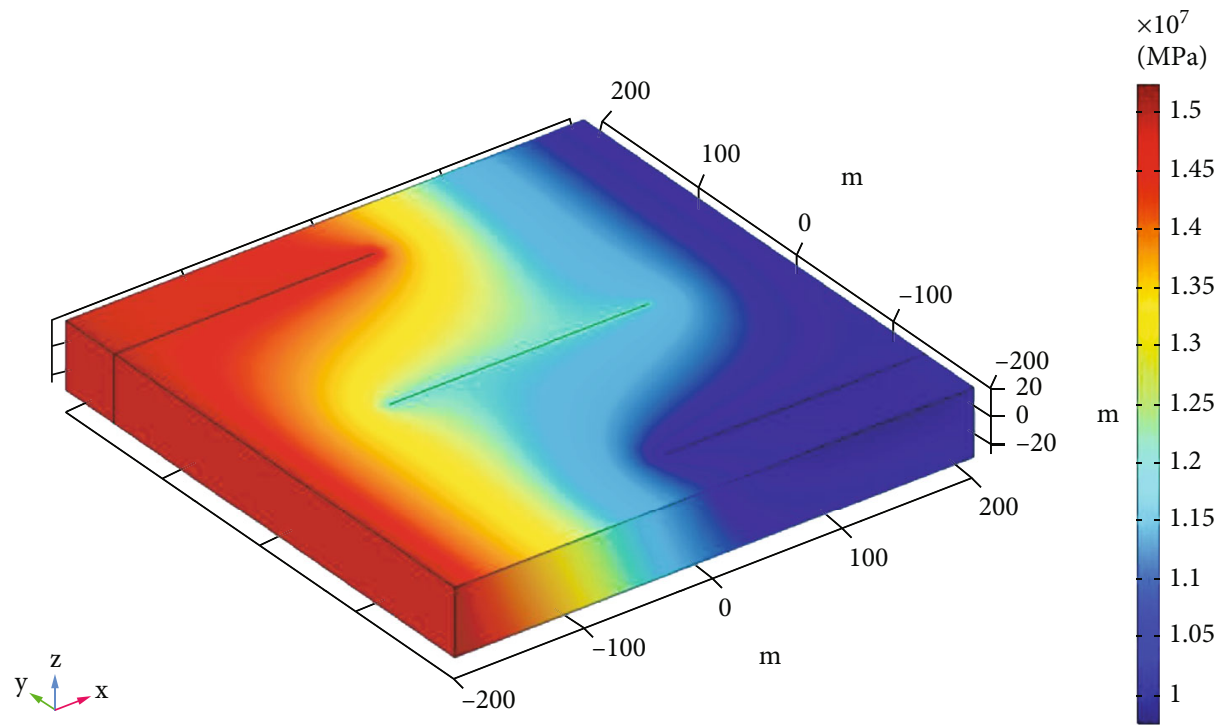

(a) Spatial distribution of pressure field of the model

$\times 10^{7}$

$(\mathrm{MPa})$

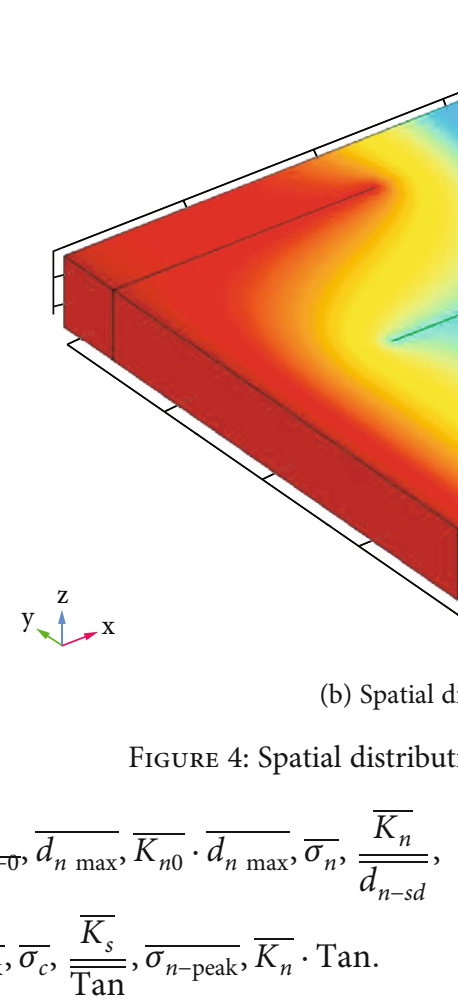

To satisfy the whole range, that is, when $\sum_{s 0} \leq \sum_{s} \leq$ $\sum_{s \text {-peak }}, 12$ similarity criteria were needed.

$$
\overline{d_{f}}, d_{\overline{n-0}}, \overline{d_{n \max }}, \overline{K_{n 0}}, \overline{\sigma_{n}}, \overline{K_{n}}, \overline{d_{n-s d}} \overline{\sigma_{s-\text { peak }}}, \overline{\sigma_{c}}, \overline{K_{s}}, \overline{\sigma_{n-\text { peak }}}, \text { Tan }
$$

Based on the study conducted by Liu et al. [20], in this study, the equation analysis method was applied to deduce and establish the similarity criterion of physical simulation of pressure-sensitive fractured anisotropic reservoirs. This similarity criterion includes a total of 55 similarity criteria number, namely, 43 similarity criteria number of pressuresensitive saturated fluid matrix and 12 similarity criteria of pressure-sensitive saturated fluid fractures, realizing the omnidirectional similarity of percolation in pressuresensitive fractured anisotropic reservoirs. Since the equation analysis method was applied for derivation, complete correspondence between microscopic and macroscopic perspectives can be achieved. Hence, this similarity criterion is applicable to design the physical simulation of pressuresensitive fractured anisotropic reservoirs.

\section{Verification by the Finite Element Model}

Under the assumption of multistratum structure, for the stress-sensitive fractured strata, the prototype and the similarity model with 100 times reduced thickness were 


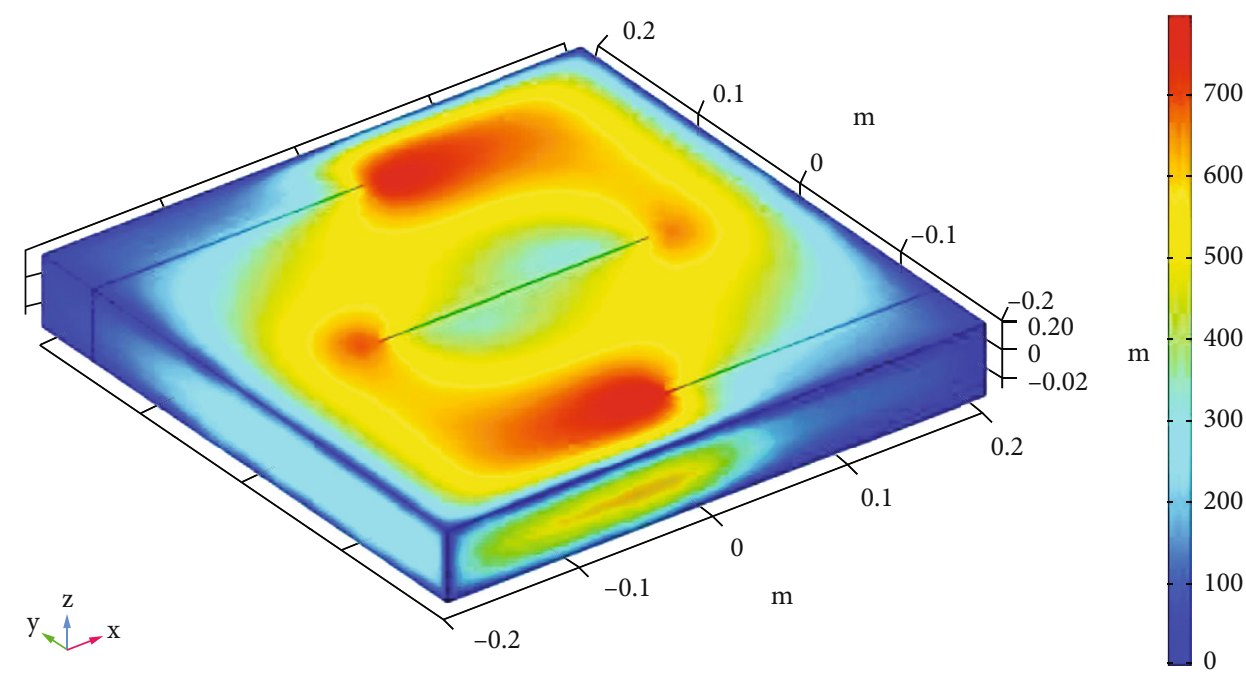

(a) Spatial distribution of effective stress of the model

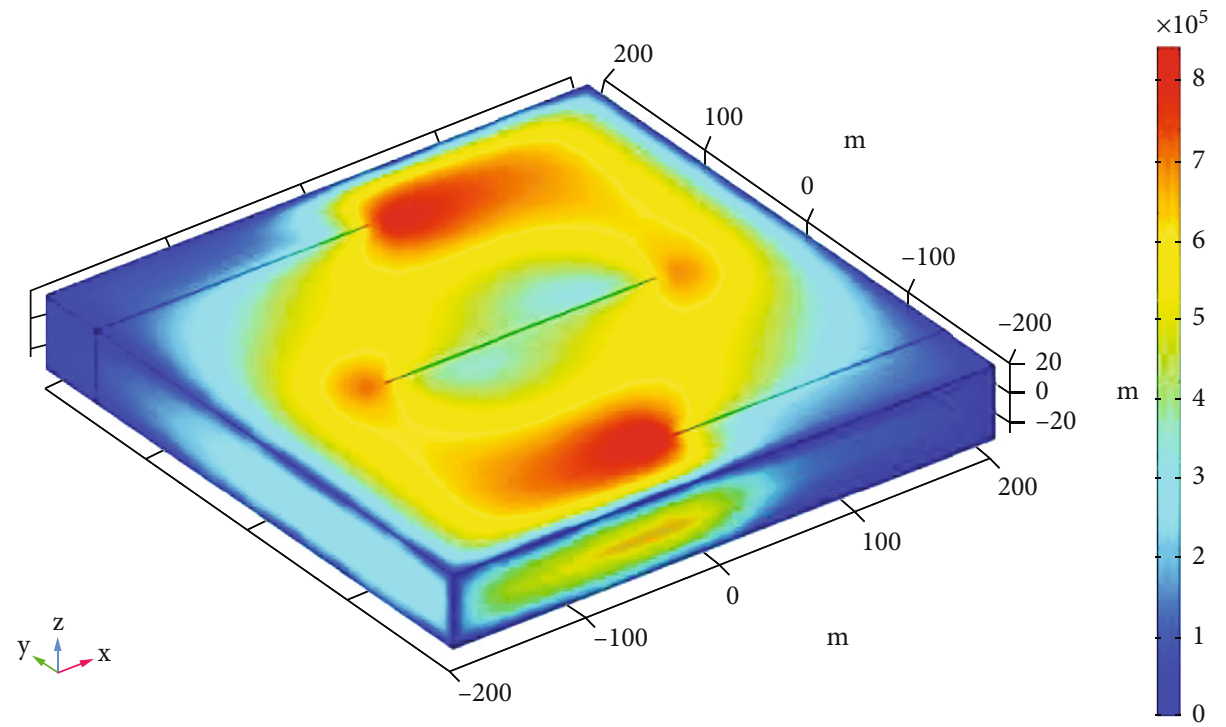

(b) Spatial distribution of effective stress of the prototype

FIgURE 5: Spatial distributions of effective stress of the prototype and the model in the $x$ direction.

established using the finite element software COMSOL Multiphysics to verify the correctness of the similarity criterion and similarity relationship.

4.1. Modeling Assumptions. The percolation of stresssensitive fractured reservoirs is a coupling process that involves multiple physical fields, including the matrix percolation field, fracture percolation field, and stress field. Therefore, it is necessary to establish a numerical model which comprehensively considers all kinds of physical fields and tries to work out the coupled solution. Since the actual situation is too complicated, the following assumptions were appropriately introduced to simplify the situation:

(1) The experimental materials are isotropic materials

(2) The percolation of the fluid follows Darcy's law
(3) The rock skeleton is compressible

(4) There is a linear relationship between the stress and strain in the matrix

(5) With slight deformation, the fluid is weakly compressible

(6) The crack wall is rough and there is no secondary filling or cementation

(7) There is no displacement at the boundary

4.2. Selection of the Prototype and Model and Parameter Setting. Firstly, a geometric model was established as shown as Figure 2. In this model, we created three cracks, of which one connected the inlet of the fluid, one was located in the middle of the medium, and the other one was connected to the outlet of the fluid. 


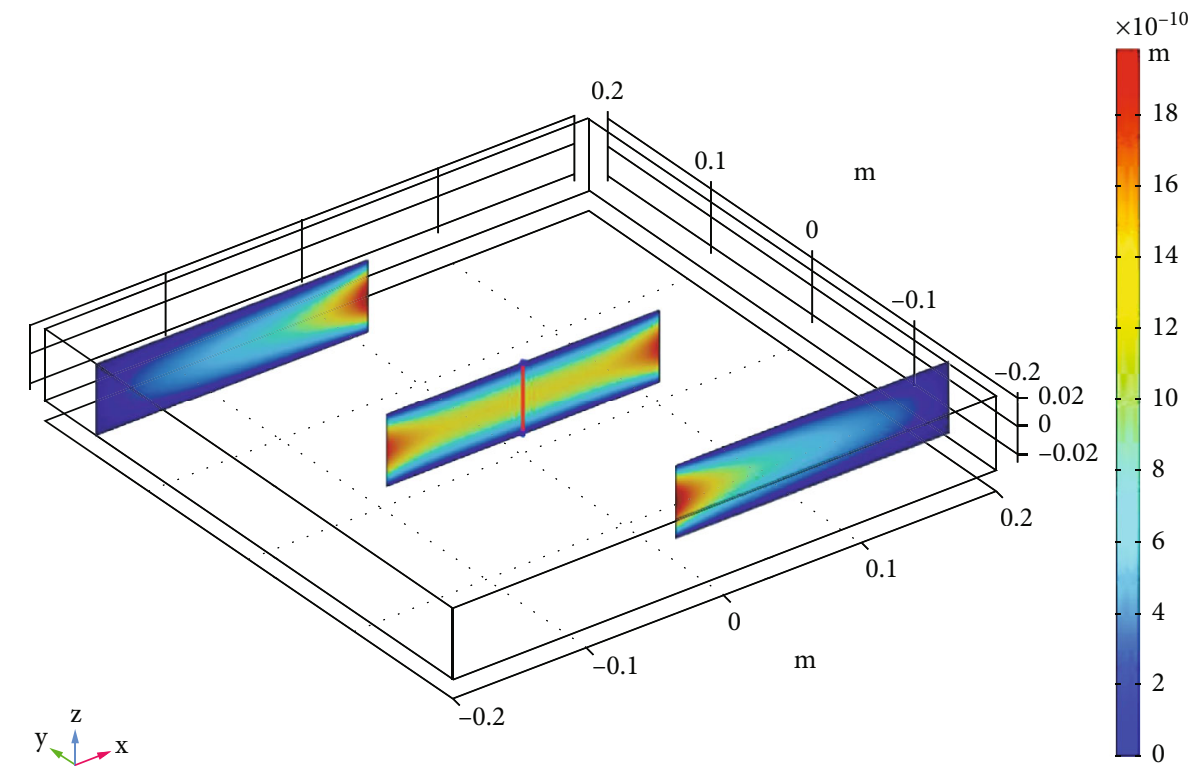

(a) Spatial distribution of fracture displacement of the model

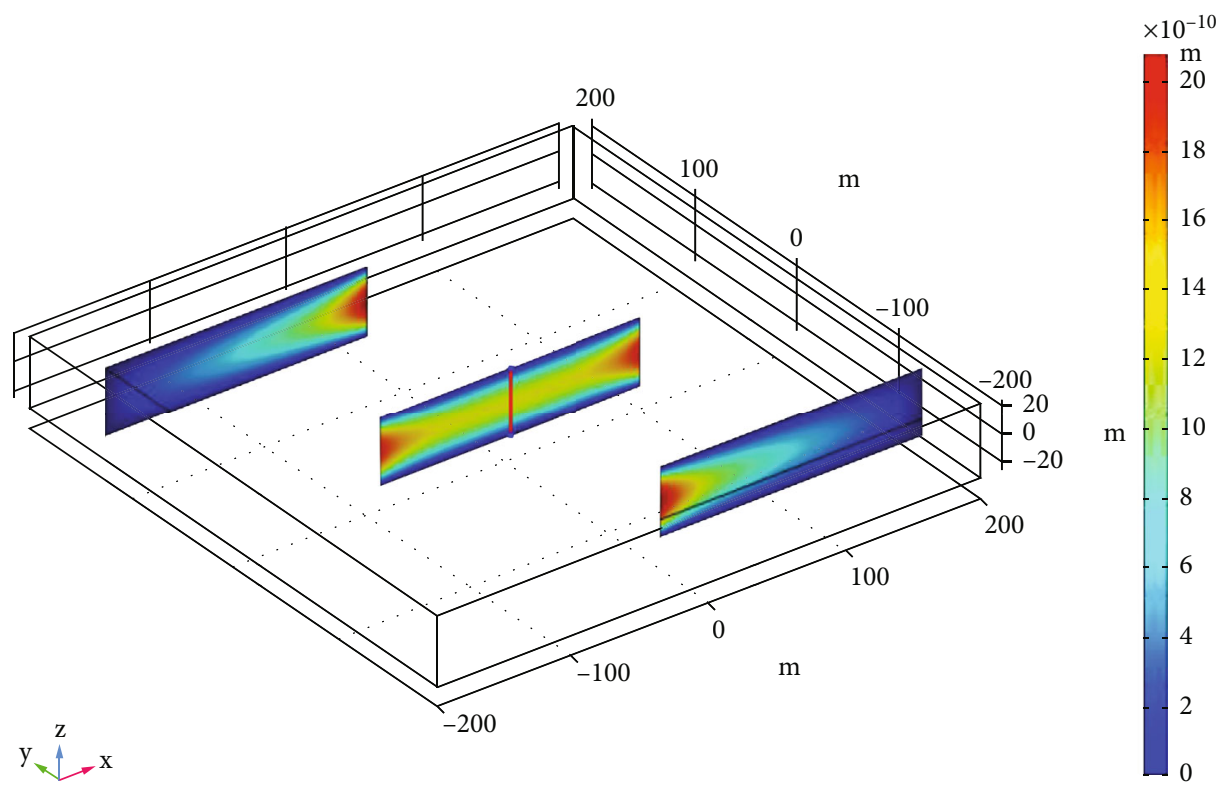

(b) Spatial distribution of fracture displacement of the prototype

FIgURE 6: Spatial distributions of fracture displacement of the prototype and the model.

4.3. Grid Generation. To guarantee consistent grid size of the model and avoid the grid scale effect caused by the difference in calculation accuracy, when establishing the model and dividing the grid, the grid scale was maintained at the same order of magnitude, namely, $1-10 \mathrm{~m}$. Figure 3 shows the grid generation of the prototype and the model. Among them, the prototype has 44914 grids and the model has 987 grids.

4.4. Data Input. Calculated according to the similarity criterion, the parameters of the prototype and the model were set as shown in Table 2 to verify the similarity of the model. In this study, the similarity parameters were mainly gained by changing the parameters relatively easy to control, e.g., model size, physical properties of the model, and the properties of the material.

\section{Verification of the Calculation Results}

Through finite element calculation, the spatial distributions of the pressure field, effective stress, and fracture displacement of the prototype and the model were obtained as shown in Figures 4-6.

By analyzing Figures 4 and 5, it is found that when the finite element calculation error is ignored, the model has a $x$ direction normal strain similar to that of the prototype. In other words, the amplitude, spatial distribution, and time history of the $x$ direction strain fields of the prototype and the 


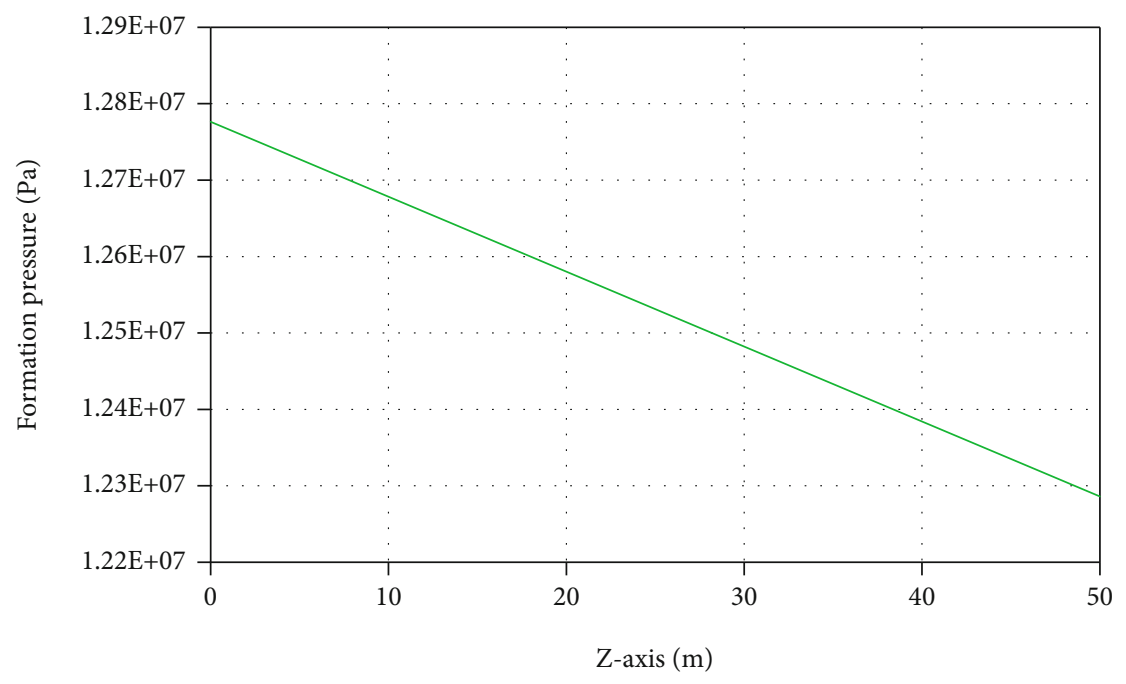

(a) Pressure distribution of the model on the red line

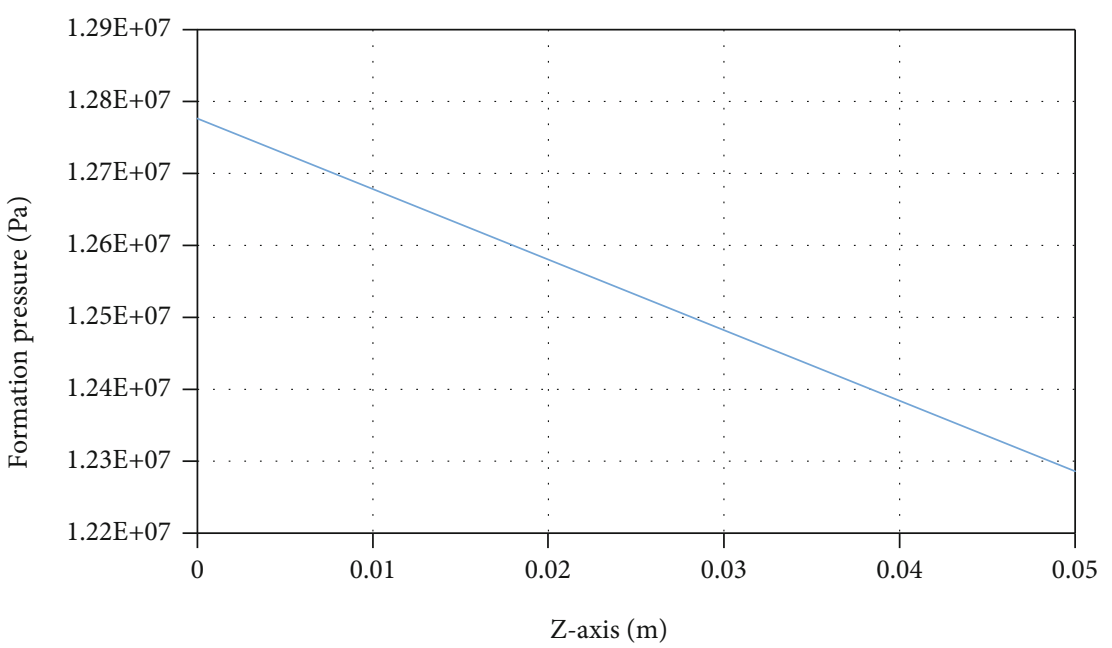

(b) Pressure distribution of the prototype on the red line

FIGURE 7: Pressure distributions of the prototype and the model.

model are consistent, which agrees with the proportional relationship (on the corresponding measuring points, the model's stress in the $x$ and $y$ directions is $1 / 1000$ of that of the prototype) theoretically deduced in Equation (24).

As shown in Figure 6, when the finite element calculation error is ignored, the fracture displacement of the model and the prototype has similar spatial distributions, which is consistent with the proportional relationship (on the corresponding measuring points, the model's stress in the $x$ direction is $1 / 1000$ of that of the prototype).

Figures 7 and 8 show the pressure distributions and spatial distributions of displacement of the prototype and the model on the red line. It can be concluded that the model and the prototype are similar, which conforms to the similarity criterion.

\section{Discussion}

This study provides a method to design the physical model for fractured reservoir pressure-sensitive simulation. The researchers can build a quantitative $3 \mathrm{D}$ model in the lab to simulate the actual reservoir. Many new coupling mechanisms can be detected by the physical method. However, the disadvantages of the similarity criteria cannot be ignored. The materials of porous medium to build the physical model seem very hard to get. Because the materials of the matrix need to meet the requirements of many aspects of the similarity criteria, such as Young's modulus, Poisson's ratio, porosity, and permeability. If the materials have some aspects of cannot meet, other parameters such as the model size must be adjusted in design of physical simulation to fit the similarity criteria.

\section{Summary and Conclusions}

In this study, the main conclusions of the cross-scale similarity modeling of the stress-sensitive fractured medium are as follows:

(1) According to the differential equations of stress-strain and fracture percolation, the similarity criterion and 


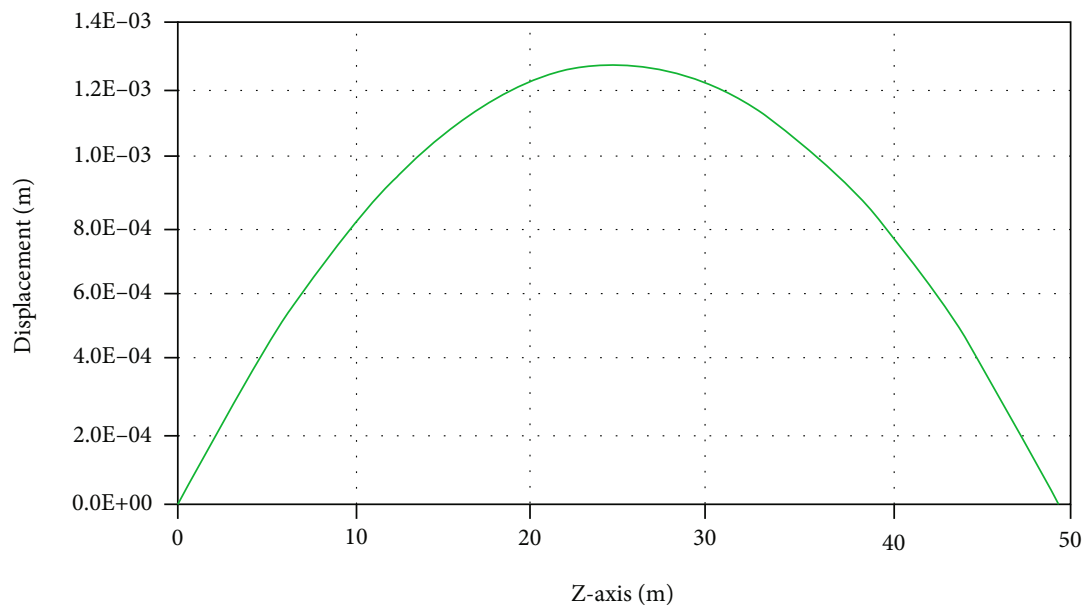

(a) Spatial distribution of displacement of the model on the red line

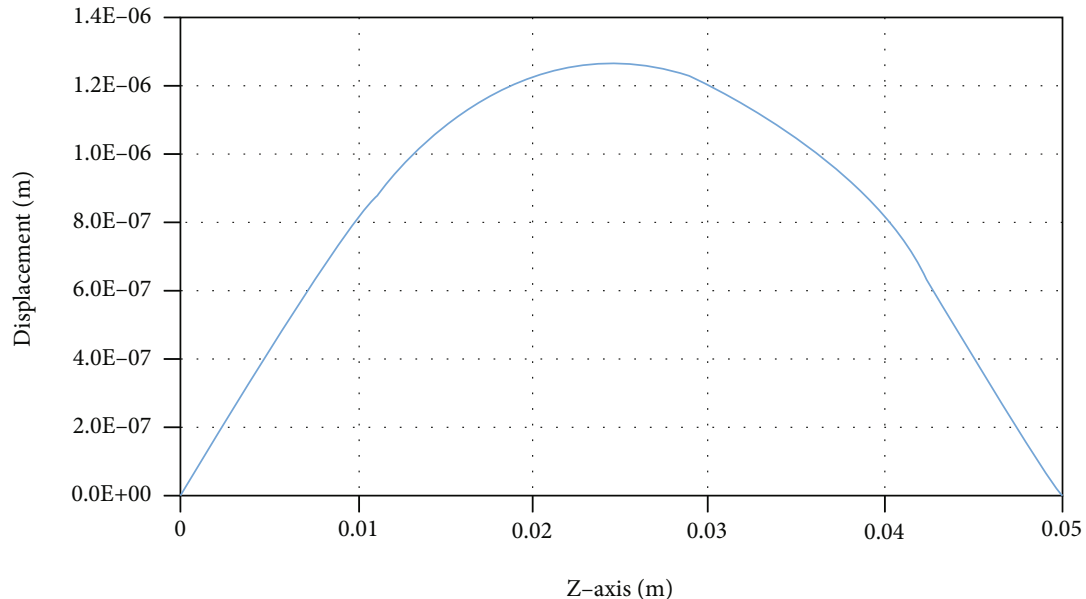

(b) Spatial distribution of displacement of the prototype on the red line

FiguRE 8: Spatial distributions of displacement of the prototype and the model.

similarity relationship obtained by dimensionless analysis is able to concurrently simulate the time history and spatial distribution of physical quantities of stress-strain and fluid pressure field

(2) To achieve the similarity of physical quantities related to the stress-strain field and the pressure field, it is only necessary to accordingly adjust the material size, percolation properties, load, and boundary conditions. The similarity between the physical model and the field size model can be realized by magnifying the geometric size $N$ times in a certain direction and adjusting the load and boundary conditions according to the similarity principle

(3) In this paper, the equation analysis method was applied to derive a similarity criterion of pressuresensitive fractured anisotropic reservoirs from abovementioned researches. Besides, the correctness of this similarity criterion and similarity relationship was verified by the finite element software, thereby laying a foundation for guiding the study of relevant physical simulation

\section{Appendix}

To derive the similarity criteria of percolation of pressuresensitive fractured reservoirs, the displacement potential function $\varphi(x, y, z)$ was introduced, and the dimensionless method was used to nondimensionalize the percolation equation of the unknown function that only contained the displacement potential function $\psi(x, y, z)$.

(1) Equilibrium equations

Equilibrium equation in the $x$ direction

$$
\begin{gathered}
c_{11} \frac{\partial^{3} \psi}{\partial x^{3}}+c_{26} \frac{\partial^{3} \psi}{\partial y^{3}}+c_{35} \frac{\partial^{3} \psi}{\partial z^{3}}+3 c_{16} \frac{\partial^{3} \psi}{\partial x^{2} \partial y}+3 c_{15} \frac{\partial^{3} \psi}{\partial x^{2} \partial z} \\
+\left(c_{12}+2 c_{66}\right) \frac{\partial^{3} \psi}{\partial x^{2} \partial y}+\left(c_{13}+2 c_{55}\right) \frac{\partial^{3} \psi}{\partial x \partial z^{2}} \\
+\left(c_{25}+2 c_{46}\right) \frac{\partial^{3} \psi}{\partial y^{2} \partial z}+\left(c_{36}+2 c_{45}\right) \frac{\partial^{3} \psi}{\partial y \partial z^{2}} \\
+2\left(c_{14}+2 c_{56}\right) \frac{\partial^{3} \psi}{\partial x \partial y \partial z}-\alpha \frac{\partial p}{\partial x}+f_{y}=0
\end{gathered}
$$


Equilibrium equation in the $y$ direction

$$
\begin{aligned}
& c_{16} \frac{\partial^{3} \psi}{\partial x^{3}}+c_{22} \frac{\partial^{3} \psi}{\partial y^{3}}+c_{34} \frac{\partial^{3} \psi}{\partial z^{3}}+3 c_{26} \frac{\partial^{3} \psi}{\partial x \partial y^{2}}+3 c_{24} \frac{\partial^{3} \psi}{\partial y^{2} \partial z} \\
& +\left(c_{12}+2 c_{66}\right) \frac{\partial^{3} \psi}{\partial x^{2} \partial y}+\left(c_{14}+2 c_{56}\right) \frac{\partial^{3} \psi}{\partial x^{2} \partial z} \\
& +\left(c_{36}+2 c_{45}\right) \frac{\partial^{3} \psi}{\partial x \partial z^{2}}+\left(c_{23}+2 c_{44}\right) \frac{\partial^{3} \psi}{\partial y \partial z^{2}} \\
& +2\left(c_{25}+2 c_{46}\right) \frac{\partial^{3} \psi}{\partial x \partial y \partial z}-\alpha \frac{\partial p}{\partial x}+f_{y}=0
\end{aligned}
$$

Equilibrium equation in the $z$ direction

$$
\begin{aligned}
& c_{15} \frac{\partial^{3} \psi}{\partial x^{3}}+c_{24} \frac{\partial^{3} \psi}{\partial y^{3}}+c_{33} \frac{\partial^{3} \psi}{\partial z^{3}}+3 c_{35} \frac{\partial^{3} \psi}{\partial x \partial z^{2}}+3 c_{34} \frac{\partial^{3} \psi}{\partial y \partial z^{2}} \\
& +\left(c_{13}+2 c_{55}\right) \frac{\partial^{3} \psi}{\partial x^{2} \partial z}+\left(c_{14}+2 c_{56}\right) \frac{\partial^{3} \psi}{\partial x^{2} \partial y} \\
& \quad+\left(c_{25}+2 c_{46}\right) \frac{\partial^{3} \psi}{\partial x \partial y^{2}}+\left(c_{23}+2 c_{44}\right) \frac{\partial^{3} \psi}{\partial y \partial z^{2}} \\
& \quad+2\left(c_{36}+2 c_{45}\right) \frac{\partial^{3} \psi}{\partial x \partial y \partial z}-\alpha \frac{\partial p}{\partial x}+f_{z}=0
\end{aligned}
$$

(2) Boundary conditions

Stress boundary equation in the $x$ direction

$$
\begin{aligned}
& n_{x}\left(c_{11} \frac{\partial^{2} \psi}{\partial x^{2}}+c_{12} \frac{\partial^{2} \psi}{\partial y^{2}}+c_{13} \frac{\partial^{2} \psi}{\partial z^{2}}+c_{14} \frac{2 \partial^{2} \psi}{\partial y \partial z}+c_{15} \frac{2 \partial^{2} \psi}{\partial x \partial z}\right. \\
& \left.+c_{16} \frac{2 \partial^{2} \psi}{\partial x \partial y}\right)+n_{y}\left(c_{14} \frac{\partial^{2} \psi}{\partial x^{2}}+c_{24} \frac{\partial^{2} \psi}{\partial y^{2}}+c_{34} \frac{\partial^{2} \psi}{\partial z^{2}}\right. \\
& \left.+c_{44} \frac{2 \partial^{2} \psi}{\partial y \partial z}+c_{45} \frac{2 \partial^{2} \psi}{\partial x \partial z}+c_{46} \frac{2 \partial^{2} \psi}{\partial x \partial y}\right)+n_{z}\left(c_{16} \frac{\partial^{2} \psi}{\partial x^{2}}\right. \\
& \left.+c_{26} \frac{\partial^{2} \psi}{\partial y^{2}}+c_{36} \frac{\partial^{2} \psi}{\partial z^{2}}+c_{46} \frac{2 \partial^{2} \psi}{\partial y \partial z}+c_{56} \frac{2 \partial^{2} \psi}{\partial x \partial z}+c_{66} \frac{2 \partial^{2} \psi}{\partial x \partial y}\right) \\
& -n_{x} \alpha p+\zeta_{x} \frac{\partial \psi}{\partial x}=P_{x}
\end{aligned}
$$

Stress boundary equation in the $y$ direction

$$
\begin{gathered}
n_{y}\left(c_{12} \frac{\partial^{2} \psi}{\partial x^{2}}+c_{22} \frac{\partial^{2} \psi}{\partial y^{2}}+c_{23} \frac{\partial^{2} \psi}{\partial z^{2}}+c_{24} \frac{2 \partial^{2} \psi}{\partial y \partial z}+c_{25} \frac{2 \partial^{2} \psi}{\partial x \partial z}\right. \\
\left.+c_{26} \frac{2 \partial^{2} \psi}{\partial x \partial y}\right)+n_{x}\left(c_{14} \frac{\partial^{2} \psi}{\partial x^{2}}+c_{24} \frac{\partial^{2} \psi}{\partial y^{2}}+c_{34} \frac{\partial^{2} \psi}{\partial z^{2}}\right.
\end{gathered}
$$

$$
\begin{aligned}
& \left.+c_{44} \frac{2 \partial^{2} \psi}{\partial y \partial z}+c_{45} \frac{2 \partial^{2} \psi}{\partial x \partial z}+c_{46} \frac{2 \partial^{2} \psi}{\partial x \partial y}\right)+n_{z}\left(c_{15} \frac{\partial^{2} \psi}{\partial x^{2}}\right. \\
& \left.+c_{25} \frac{\partial^{2} \psi}{\partial y^{2}}+c_{35} \frac{\partial^{2} \psi}{\partial z^{2}}+c_{45} \frac{2 \partial^{2} \psi}{\partial y \partial z}+c_{55} \frac{2 \partial^{2} \psi}{\partial x \partial z}+c_{56} \frac{2 \partial^{2} \psi}{\partial x \partial y}\right) \\
& -n_{y} \alpha p+\zeta_{y} \frac{\partial \psi}{\partial y}=P_{y}
\end{aligned}
$$

Stress boundary equation in the $z$ direction

$$
\begin{aligned}
& n_{z}\left(c_{13} \frac{\partial^{2} \psi}{\partial x^{2}}+c_{23} \frac{\partial^{2} \psi}{\partial y^{2}}+c_{33} \frac{\partial^{2} \psi}{\partial z^{2}}+c_{34} \frac{2 \partial^{2} \psi}{\partial y \partial z}+c_{35} \frac{2 \partial^{2} \psi}{\partial x \partial z}\right. \\
& \left.+c_{36} \frac{2 \partial^{2} \psi}{\partial x \partial y}\right)+n_{x}\left(c_{15} \frac{\partial^{2} \psi}{\partial x^{2}}+c_{25} \frac{\partial^{2} \psi}{\partial y^{2}}+c_{35} \frac{\partial^{2} \psi}{\partial z^{2}}\right. \\
& \left.+c_{45} \frac{2 \partial^{2} \psi}{\partial y \partial z}+c_{55} \frac{2 \partial^{2} \psi}{\partial x \partial z}+c_{56} \frac{2 \partial^{2} \psi}{\partial x \partial y}\right)+n_{y}\left(c_{16} \frac{\partial^{2} \psi}{\partial x^{2}}\right. \\
& \left.\quad+c_{26} \frac{\partial^{2} \psi}{\partial y^{2}}+c_{36} \frac{\partial^{2} \psi}{\partial z^{2}}+c_{46} \frac{2 \partial^{2} \psi}{\partial y \partial z}+c_{56} \frac{2 \partial^{2} \psi}{\partial x \partial z}+c_{66} \frac{2 \partial^{2} \psi}{\partial x \partial y}\right) \\
& \quad-n_{z} \alpha p+\zeta_{z} \frac{\partial \psi}{\partial z}=P_{z}
\end{aligned}
$$

The displacement boundary conditions in the $x, y$, and $z$ directions are as follows:

$$
\begin{gathered}
u_{\Gamma}=\frac{\partial \psi}{\partial x} \\
v_{\Gamma}=\frac{\partial \psi}{\partial y}, \\
w_{\Gamma}=\frac{\partial \psi}{\partial z}
\end{gathered}
$$

To nondimensionalize Equations (A.1)-(A.9), the following scales were introduced: $A$ is the unit length in the $x$ direction, $B$ is the unit length in the $y$ direction, and $C$ is the unit length in the $z$ direction, with $[L]$ being the dimension. $\psi_{o}$ is the unit potential equation, with $\left[L^{2}\right]$ being the dimension. $f_{x 0}, f_{y 0}$, and $f_{z 0}$ refer to the unit physical strength, with $\left[M L^{-2} T^{-2}\right]$ being the dimension. $P_{0}$ denotes the unit pressure, with $\left[L^{-1} M T^{2}\right]$ being the dimension. $p_{x 0}$, $p_{y 0}$, and $p_{z 0}$ represent the unit boundary stress, with $\left[M L^{-2}\right.$ $\left.T^{-2}\right]$ being the dimension.

Substituting the above unit parameters into Equations (A.1)-(A.9), the following dimensionless equations were gained:

Dimensionless basic equations of elasticity: 
(1) Equilibrium equations

(1) Dimensionless equilibrium equation in the $x$ direction

$$
\begin{aligned}
\frac{c_{11}}{f_{x 0}} & \frac{\psi_{0}}{A^{3}} \frac{\partial^{3} \Psi}{\partial X^{3}}+\frac{c_{26}}{f_{x 0}} \frac{\psi_{0}}{B^{3}} \frac{\partial^{3} \Psi}{\partial Y^{3}}+\frac{c_{35}}{f_{x 0}} \frac{\psi_{0}}{C^{3}} \frac{\partial^{3} \Psi}{\partial Z^{3}}+\frac{3 c_{16}}{f_{x 0}} \frac{\psi_{0}}{A^{2} B} \frac{\partial^{3} \Psi}{\partial X^{2} \partial Y} \\
+ & \frac{3 c_{15}}{f_{x 0}} \frac{\psi_{0}}{A^{2} C} \frac{\partial^{3} \Psi}{\partial X^{2} \partial Z}+\frac{\left(c_{12}+2 c_{66}\right)}{f_{x 0}} \frac{\psi_{0}}{A B^{2}} \frac{\partial^{3} \Psi}{\partial X \partial Y^{2}} \\
+ & \frac{\left(c_{13}+2 c_{55}\right)}{f_{x 0}} \frac{\psi_{0}}{A C^{2}} \frac{\partial^{3} \Psi}{\partial X \partial Z^{2}}+\frac{\left(c_{25}+2 c_{46}\right)}{f_{x 0}} \frac{\psi_{0}}{B^{2} C} \frac{\partial^{3} \Psi}{\partial Y^{2} \partial Z} \\
+ & \frac{\left(c_{36}+2 c_{45}\right)}{f_{x 0}} \frac{\psi_{0}}{B C^{2}} \frac{\partial^{3} \Psi}{\partial Y \partial Z^{2}}+\frac{2\left(c_{14}+2 c_{56}\right)}{f_{x 0}} \frac{\psi_{0}}{A B C} \frac{\partial^{3} \Psi}{\partial X \partial Y \partial Z} \\
& -\alpha \frac{p_{0}}{f_{x 0}} \frac{\partial P}{\partial X}+F_{x}=0
\end{aligned}
$$

(2) Dimensionless equilibrium equation in the $y$ direction

$$
\begin{gathered}
\frac{c_{16}}{f_{y 0}} \frac{\psi_{0}}{A^{3}} \frac{\partial^{3} \Psi}{\partial X^{3}}+\frac{c_{22}}{f_{y 0}} \frac{\psi_{0}}{B^{3}} \frac{\partial^{3} \Psi}{\partial Y^{3}}+\frac{c_{34}}{f_{y 0}} \frac{\psi_{0}}{C^{3}} \frac{\partial^{3} \Psi}{\partial Z^{3}} \\
+\frac{3 c_{26}}{f_{y 0}} \frac{\psi_{0}}{A B^{2}} \frac{\partial^{3} \Psi}{\partial X \partial Y^{2}}+\frac{3 c_{24}}{f_{y 0}} \frac{\psi_{0}}{B^{2} C} \frac{\partial^{3} \Psi}{\partial X^{2} \partial Z} \\
+\frac{\left(c_{12}+2 c_{66}\right)}{f_{y 0}} \frac{\psi_{0}}{A^{2} B} \frac{\partial^{3} \Psi}{\partial X^{2} \partial Y}+\frac{\left(c_{14}+2 c_{56}\right)}{f_{y 0}} \\
+\frac{\psi_{0}}{A^{2} C} \frac{\partial^{3} \Psi}{\partial X^{2} \partial Z}+\frac{\left(c_{36}+2 c_{45}\right)}{f_{y 0}} \frac{\psi_{0}}{A C^{2}} \frac{\partial^{3} \Psi}{\partial X \partial Z^{2}} \\
+\frac{\left(c_{23}+2 c_{44}\right)}{f_{y 0}} \frac{\psi_{0}}{B C^{2}} \frac{\partial^{3} \Psi}{\partial Y \partial Z^{2}}+\frac{2\left(c_{25}+2 c_{46}\right)}{f_{y 0}} \\
\cdot \frac{\psi_{0}}{A B C} \frac{\partial^{3} \Psi}{\partial X \partial Y \partial Z}-\alpha \frac{p_{0}}{f_{y 0} B} \frac{\partial P}{\partial Y}+F_{y}=0
\end{gathered}
$$

(3) Dimensionless equilibrium equation in the $z$ direction

$$
\begin{gathered}
\frac{c_{15}}{f_{z 0}} \frac{\psi_{0}}{A^{3}} \frac{\partial^{3} \Psi}{\partial X^{3}}+\frac{c_{24}}{f_{z 0}} \frac{\psi_{0}}{B^{3}} \frac{\partial^{3} \Psi}{\partial Y^{3}}+\frac{c_{33}}{f_{z 0}} \frac{\psi_{0}}{C^{3}} \frac{\partial^{3} \Psi}{\partial Z^{3}} \\
+\frac{3 c_{35}}{f_{z 0}} \frac{\psi_{0}}{A C^{2}} \frac{\partial^{3} \Psi}{\partial X \partial Z^{2}}+\frac{3 c_{34}}{f_{z 0}} \frac{\psi_{0}}{B C^{2}} \frac{\partial^{3} \Psi}{\partial Y \partial Z^{2}} \\
+\frac{\left(c_{13}+2 c_{55}\right)}{f_{z 0}} \frac{\psi_{0}}{A^{2} C} \frac{\partial^{3} \Psi}{\partial X^{2} \partial Y}+\frac{\left(c_{14}+2 c_{56}\right)}{f_{z 0}} \\
\cdot \frac{\psi_{0}}{A^{2} B} \frac{\partial^{3} \Psi}{\partial X^{2} \partial Y}+\frac{\left(c_{25}+2 c_{46}\right)}{f_{z 0}} \frac{\psi_{0}}{A B^{2}} \frac{\partial^{3} \Psi}{\partial X \partial Y^{2}} \\
+\frac{\left(c_{23}+2 c_{44}\right)}{f_{z 0}} \frac{\psi_{0}}{B C^{2}} \frac{\partial^{3} \Psi}{\partial Y \partial Z^{2}}+\frac{2\left(c_{36}+2 c_{45}\right)}{f_{z 0}} \\
\cdot \frac{\psi_{0}}{A B C} \frac{\partial^{3} \Psi}{\partial X \partial Y \partial Z}-\alpha \frac{p_{0}}{f_{z 0} C} \frac{\partial P}{\partial Z}+F_{z}=0
\end{gathered}
$$

(2) Dimensionless boundary conditions
(1) Dimensionless boundary stress in the $x$ direction

$$
\begin{aligned}
& n_{x}\left(\frac{c_{11}}{p_{x 0}} \frac{\psi_{0}}{A^{2}} \frac{\partial^{2} \Psi}{\partial X^{2}}+\frac{c_{26}}{p_{x 0}} \frac{\psi_{0}}{B^{2}} \frac{\partial^{2} \Psi}{\partial Y^{2}}+\frac{c_{35}}{p_{x 0}} \frac{\psi_{0}}{C^{2}} \frac{\partial^{2} \Psi}{\partial Z^{2}}\right. \\
& \left.+\frac{2 c_{14}}{p_{x 0}} \frac{\psi_{0}}{B C} \frac{\partial^{2} \Psi}{\partial Y \partial Z}+\frac{2 c_{15}}{p_{x 0}} \frac{\psi_{0}}{A C} \frac{\partial^{2} \Psi}{\partial X \partial Z}+\frac{2 c_{16}}{p_{x 0}} \frac{\psi_{0}}{A B} \frac{\partial^{2} \Psi}{\partial X \partial Y}\right) \\
& +n_{y}\left(\frac{c_{14}}{p_{x 0}} \frac{\psi_{0}}{A^{2}} \frac{\partial^{2} \Psi}{\partial X^{2}}+\frac{c_{24}}{p_{x 0}} \frac{\psi_{0}}{B^{2}} \frac{\partial^{2} \Psi}{\partial Y^{2}}+\frac{c_{34}}{p_{x 0}} \frac{\psi_{0}}{C^{2}} \frac{\partial^{2} \Psi}{\partial Z^{2}}\right. \\
& \left.+\frac{2 c_{44}}{p_{x 0}} \frac{\psi_{0}}{B C} \frac{\partial^{2} \Psi}{\partial Y \partial Z}+\frac{2 c_{45}}{p_{x 0}} \frac{\psi_{0}}{A C} \frac{\partial^{2} \Psi}{\partial X \partial Z}+\frac{2 c_{46}}{p_{x 0}} \frac{\psi_{0}}{A B} \frac{\partial^{2} \Psi}{\partial X \partial Y}\right) \\
& +n_{z}\left(\frac{c_{16}}{p_{x 0}} \frac{\psi_{0}}{A^{2}} \frac{\partial^{2} \Psi}{\partial X^{2}}+\frac{c_{26}}{p_{x 0}} \frac{\psi_{0}}{B^{2}} \frac{\partial^{2} \Psi}{\partial Y^{2}}+\frac{c_{36}}{p_{x 0}} \frac{\psi_{0}}{C^{2}} \frac{\partial^{2} \Psi}{\partial Z^{2}}\right. \\
& \left.+\frac{2 c_{46}}{p_{x 0}} \frac{\psi_{0}}{B C} \frac{\partial^{2} \Psi}{\partial Y \partial Z}+\frac{2 c_{56}}{p_{x 0}} \frac{\psi_{0}}{A C} \frac{\partial^{2} \Psi}{\partial X \partial Z}+\frac{2 c_{66}}{p_{x 0}} \frac{\psi_{0}}{A B} \frac{\partial^{2} \Psi}{\partial X \partial Y}\right) \\
& +n_{x} \alpha \frac{p_{0}}{p_{x 0}} P+\zeta_{x} \frac{1}{p_{x 0} A} \frac{\partial \Psi}{\partial X}=P_{x}
\end{aligned}
$$

(2) Dimensionless boundary stress in the $y$ direction

$$
\begin{aligned}
& n_{y}\left(\frac{c_{12}}{p_{y 0}} \frac{\psi_{0}}{A^{2}} \frac{\partial^{2} \Psi}{\partial X^{2}}+\frac{c_{22}}{p_{y 0}} \frac{\psi_{0}}{B^{2}} \frac{\partial^{2} \Psi}{\partial Y^{2}}+\frac{c_{23}}{p_{y 0}} \frac{\psi_{0}}{C^{2}} \frac{\partial^{2} \Psi}{\partial Z^{2}}\right. \\
& \left.+\frac{2 c_{24}}{p_{y 0}} \frac{\psi_{0}}{B C} \frac{\partial^{2} \Psi}{\partial Y \partial Z}+\frac{2 c_{25}}{p_{y 0}} \frac{\psi_{0}}{A C} \frac{\partial^{2} \Psi}{\partial X \partial Z}+\frac{2 c_{26}}{p_{y 0}} \frac{\psi_{0}}{A B} \frac{\partial^{2} \Psi}{\partial X \partial Y}\right) \\
& +n_{x}\left(\frac{c_{14}}{p_{y 0}} \frac{\psi_{0}}{A^{2}} \frac{\partial^{2} \Psi}{\partial X^{2}}+\frac{c_{24}}{p_{y 0}} \frac{\psi_{0}}{B^{2}} \frac{\partial^{2} \Psi}{\partial Y^{2}}+\frac{c_{34}}{p_{y 0}} \frac{\psi_{0}}{C^{2}} \frac{\partial^{2} \Psi}{\partial Z^{2}}\right. \\
& \left.+\frac{2 c_{44}}{p_{y 0}} \frac{\psi_{0}}{B C} \frac{\partial^{2} \Psi}{\partial Y \partial Z}+\frac{2 c_{45}}{p_{y 0}} \frac{\psi_{0}}{A C} \frac{\partial^{2} \Psi}{\partial X \partial Z}+\frac{2 c_{46}}{p_{y 0}} \frac{\psi_{0}}{A B} \frac{\partial^{2} \Psi}{\partial X \partial Y}\right) \\
& +n_{z}\left(\frac{c_{15}}{p_{y 0}} \frac{\psi_{0}}{A^{2}} \frac{\partial^{2} \Psi}{\partial X^{2}}+\frac{c_{25}}{p_{y 0}} \frac{\psi_{0}}{B^{2}} \frac{\partial^{2} \Psi}{\partial Y^{2}}+\frac{c_{35}}{p_{y 0}} \frac{\psi_{0}}{C^{2}} \frac{\partial^{2} \Psi}{\partial Z^{2}}\right. \\
& \left.+\frac{2 c_{45}}{p_{y 0}} \frac{\psi_{0}}{B C} \frac{\partial^{2} \Psi}{\partial Y \partial Z}+\frac{2 c_{55}}{p_{y 0}} \frac{\psi_{0}}{A C} \frac{\partial^{2} \Psi}{\partial X \partial Z}+\frac{2 c_{56}}{p_{y 0}} \frac{\psi_{0}}{A B} \frac{\partial^{2} \Psi}{\partial X \partial Y}\right) \\
& +n_{y} \alpha \frac{p_{0}}{p_{y 0}} P+\zeta_{y} \frac{1}{p_{y 0} B} \frac{\partial \Psi}{\partial Y}=P_{y}
\end{aligned}
$$

(3) Dimensionless boundary stress in the $z$ direction

$$
n_{z}\left(\frac{c_{13}}{p_{z 0}} \frac{\psi_{0}}{A^{2}} \frac{\partial^{2} \Psi}{\partial X^{2}}+\frac{c_{23}}{p_{z 0}} \frac{\psi_{0}}{B^{2}} \frac{\partial^{2} \Psi}{\partial Y^{2}}+\frac{c_{33}}{p_{z 0}} \frac{\psi_{0}}{C^{2}} \frac{\partial^{2} \Psi}{\partial Z^{2}}\right.
$$




$$
\begin{aligned}
& \left.+\frac{2 c_{34}}{p_{z 0}} \frac{\psi_{0}}{B C} \frac{\partial^{2} \Psi}{\partial Y \partial Z}+\frac{2 c_{35}}{p_{z 0}} \frac{\psi_{0}}{A C} \frac{\partial^{2} \Psi}{\partial X \partial Z}+\frac{2 c_{36}}{p_{z 0}} \frac{\psi_{0}}{A B} \frac{\partial^{2} \Psi}{\partial X \partial Y}\right) \\
& +n_{x}\left(\frac{c_{15}}{p_{z 0}} \frac{\psi_{0}}{A^{2}} \frac{\partial^{2} \Psi}{\partial X^{2}}+\frac{c_{25}}{p_{z 0}} \frac{\psi_{0}}{B^{2}} \frac{\partial^{2} \Psi}{\partial Y^{2}}+\frac{c_{35}}{p_{z 0}} \frac{\psi_{0}}{C^{2}} \frac{\partial^{2} \Psi}{\partial Z^{2}}\right. \\
& \left.+\frac{2 c_{45}}{p_{z 0}} \frac{\psi_{0}}{B C} \frac{\partial^{2} \Psi}{\partial Y \partial Z}+\frac{2 c_{55}}{p_{z 0}} \frac{\psi_{0}}{A C} \frac{\partial^{2} \Psi}{\partial X \partial Z}+\frac{2 c_{56}}{p_{z 0}} \frac{\psi_{0}}{A B} \frac{\partial^{2} \Psi}{\partial X \partial Y}\right) \\
& +n_{y}\left(\frac{c_{16}}{p_{z 0}} \frac{\psi_{0}}{A^{2}} \frac{\partial^{2} \Psi}{\partial X^{2}}+\frac{c_{26}}{p_{z 0}} \frac{\psi_{0}}{B^{2}} \frac{\partial^{2} \Psi}{\partial Y^{2}}+\frac{c_{36}}{p_{z 0}} \frac{\psi_{0}}{C^{2}} \frac{\partial^{2} \Psi}{\partial Z^{2}}\right. \\
& \left.+\frac{2 c_{46}}{p_{z 0}} \frac{\psi_{0}}{B C} \frac{\partial^{2} \Psi}{\partial Y \partial Z}+\frac{2 c_{56}}{p_{z 0}} \frac{\psi_{0}}{A C} \frac{\partial^{2} \Psi}{\partial X \partial Z}+\frac{2 c_{66}}{p_{z 0}} \frac{\psi_{0}}{A B} \frac{\partial^{2} \Psi}{\partial X \partial Y}\right) \\
& -n_{z} \alpha \frac{p_{0}}{p_{z 0}} P+\zeta_{z} \frac{1}{p_{z 0} C} \frac{\partial \Psi}{\partial Z}=P_{z}
\end{aligned}
$$

Zero-dimension displacement boundaries in the $x, y$, and $z$ directions are expressed as follows:

$$
\begin{aligned}
U_{\Gamma} & =\frac{\partial \Psi}{\partial X} \frac{\psi_{0} u_{0}}{A}, \\
V_{\Gamma} & =\frac{\partial \Psi}{\partial X} \frac{\psi_{0} v_{0}}{B} \\
W_{\Gamma} & =\frac{\partial \Psi}{\partial X} \frac{\psi_{0} u_{0}}{C},
\end{aligned}
$$

where $\Psi=\psi / \psi_{0}$ denotes the zero-dimension potential equation.

$X=x / A, Y=y / B$, and $Z=z / C$ refer to the zerodimension lengths in the $x, y$, and $z$ directions, respectively.

$P=p / p_{0}$ represents the zero-dimension pressure.

$P_{x}=p_{x} / p_{x 0}, P_{y}=p_{y} / p_{y 0}$, and $P_{z}=p_{z} / p_{z 0}$ stand for the zero-dimension boundary stress.

$F_{x}=f_{x} / f_{x 0}, F_{y}=f_{y} / f_{y 0}$, and $F_{z}=f_{z} / f_{z 0}$ denote the zerodimension physical strength.

$U_{\Gamma}=u / A, V_{\Gamma}=v / B$, and $W_{\Gamma}=w / C$ refer to the zerodimension displacement.

Based on the above derivation, the similar criteria for pressure-sensitive medium were obtained. For instance, $X$, $Y, Z, P, P_{x}, P_{y}, P_{z}, F_{x}, F_{y}, F_{z}, U_{\Gamma}, V_{\Gamma}$, and $W_{\Gamma}$ are also parameters of the potential function $\Psi$. According to the second similarity principle, if two models have the same similarity criterion and the same number of coefficients, their potential functions are also the same.

\section{Data Availability}

All data, models, and code generated or used during the study appear in the submitted article.

\section{Conflicts of Interest}

The authors declare that they have no conflicts of interest.

\section{Acknowledgments}

The writers greatly appreciate the financial support of the National Natural Science Foundation of China (Grant No. 51804039) and PetroChina Innovation Foundation (Grant No. 2017D-5007-0311).

\section{References}

[1] Y. H. Bai and J. F. Zhou, "The advance in the scaling criteria of physical simulation for the complex flows system in reservoir," Advances in Mechanics, vol. 39, no. 1, pp. 58-68, 2009.

[2] Z. P. Ding and Y. G. Qu, Similarity Criterion for Quantitative Physical Simulation of Constant Pressure Production in Water Drive Sandstone Reservoirs, The Chinese Society of Theoretical and Applied Mechanics, 2013.

[3] M. Gu, P. Guo, D. H. Tian, and Z. H. Wang, "Study on similarity criteria of physical modeling for displacement of oil by water," Special Oil \& Gas Reservoirs, vol. 23, no. 1, pp. 123126, 2016.

[4] X. Y. Kong and F. L. Chen, "Similar theory, similar rule and data conversion software for water drive modeling," Petroleum Exploration and Development, vol. 6, pp. 56-60, 1997.

[5] Y. Q. Li, Physical and Numerical Simulation of Water Flooding with Plane Heterogeneous Model, Northeast Petroleum University, 1998.

[6] Y. H. Bai, J. C. Li, and J. F. Zhou, "Sensitivity analysis of similar parameters in physical simulation of water-oil two-phase flow, science in China (Series E): engineering science materials," Science, vol. 7, no. 7, pp. 91-102, 2005.

[7] R. H. Shen, W. Xiong, and S. S. Gao, "Similarity theory of waterflooding experiment in low permeability cores," Rock and Soil Mechanics, vol. 3, pp. 138-142, 2012.

[8] Q. Teng, Z. M. Yang, X. W. Liu, Xiong S. C., Yu R. Z., and Feng P., "Derivation and application of similarity criterion for physical simulation of water displacement in extra-low permeability reservoir," Science \& Technology Review, vol. 9, pp. 40-45, 2013.

[9] X. W. Shi, Z. M. Yang, X. L. Duan et al., "Study on similarity theory of water flooding oil in low permeability carbonate reservoir," Petroleum Geology and Recovery Efficiency, vol. 25, no. 1, pp. 86-93, 2018.

[10] Z. P. Ding, Y. T. Liu, Y. G. Qu, Zhang Y., and Ao K., "Similarness criterion for three-dimensional quantitative physical simulation of water flooding in fractured reservoirs," in Innovation and Practice of Seepage Mechanics and Engineering - Proceedings of the 11th National Symposium on Seepage Mechanics, pp. 160-164, Chong Qing, 2011.

[11] B. B. Wang, Study on Similar Criterion of Steam Flooding in Gudao No.2 Middle Reservoir, China University of Petroleum, 2010.

[12] T. Z. Yu, "Physical simulation and numerical simulation of chemical flooding in horizontal Wells," Lithologic Reservoirs, vol. 25, no. 5, pp. 104-108, 2013.

[13] Y. Bai, J. Zhou, and Q. Li, "Designing of partial similarity models and evaluation method in polymer flooding experiment," Transport in Porous Media, vol. 75, no. 3, pp. 401412, 2008.

[14] Y. Bai, J. Li, J. Zhou, and Q. Li, "Sensitivity analysis of the dimensionless parameters in scaling a polymer flooding 
reservoir," Transport in Porous Media, vol. 73, no. 1, pp. 2137,2008

[15] X. S. Li, Y. Wang, G. Li, and Y. Zhang, "Experimental investigations into gas production behaviors from methane hydrate with different methods in a cubic hydrate simulator," Energy \& Fuels, vol. 26, no. 2, pp. 1124-1134, 2012.

[16] Y. Wang, C. G. Xu, X. S. Li, G. Li, and Z. Y. Chen, "Similarity analysis in scaling a gas hydrates reservoir," Energies, vol. 6, no. 5, pp. 2468-2480, 2013.

[17] Z. Sun, X. Li, W. Liu, T. Zhang, M. He, and H. Nasrabadi, "Molecular dynamics of methane flow behavior through realistic organic nanopores under geologic shale condition: pore size and kerogen types," Chemical Engineering Journal, vol. 398, article 124341, 2020.

[18] Z. Sun, J. Shi, K. Wu, T. Zhang, D. Feng, and X. Li, "Effect of pressure-propagation behavior on production performance: implication for advancing low-permeability coalbed-methane recovery," SPE Journal, vol. 24, no. 2, pp. 681-697, 2019.

[19] S. Gu, Y. Liu, and Z. Chen, "Numerical study of dynamic fracture aperture during production of pressure- sensitive reservoirs," International Journal of Rock Mechanics \& Mining Sciences, vol. 70, pp. 229-239, 2014.

[20] Y. T. Liu, W. K. Zheng, P. Yu, and J. L. Wu, Simulation Method and Model System of Rock Pressure-Sensitive Fracture, CHN.Patent, CN105716955A, 2016. 